\title{
Opt-Outs, Opt-Ins, Opt-Arounds? Eine Analyse der Differenzierungsrealität im Raum der Freiheit, der Sicherheit und des Rechts
}

\author{
Funda Tekin*
}

Das Konzept der differenzierten Integration in der Europäischen Union ist Gegenstand einer wissenschaftlichen und politischen Debatte, deren Konjunkturschwankungen durch Vertragsreformschritte, Erweiterungsrunden und Krisen bedingt sind. So hat die Frage nach einem Europa der mehreren Geschwindigkeiten, einem Kerneuropa oder einem Europa mit variabler Geometrie seit dem Beginn der Finanzkrise im Allgemeinen und besonders seit dem Beschluss zum Fiskalpakt auf dem Gipfel des Europäischen Rates vom 9. Dezember 2011 Hochkonjunktur. ${ }^{1}$ Die deutsche Bundeskanzlerin Angela Merkel hat erst im Juni 2012 der Europäischen Union mit Blick auf den Euro unterschiedliche Geschwindigkeiten attestiert und weiterhin festgestellt, dass ,,wir [...] nicht deshalb stehen bleiben [dürfen], weil der eine oder andere noch nicht mitgehen will“ “2 Das Neue am aktuellen Diskurs ist jedoch, dass diese Integrationsform bei der „eine bestimmte Gruppe von Mitgliedstaaten nicht denselben Unionsregeln unterliegt wie die übrigen Mitgliedstaaten" ${ }^{\text {"3 }}$ nicht nur als mögliche Strategie zur Konsolidierung von Vertiefung und Erweiterung der Europäischen Union diskutiert wird. ${ }^{4}$ Sondern ein differenziertes Vorgehen scheint angesichts der Heterogenität in der Europäischen Union bezüglich der wirtschaftlichen Fähigkeit, den aktuellen Integrationsstand in der Wirtschafts- und Währungsunion zu bewahren, und des politischen Willens, mehr Souveränitätsrechte in sensiblen Politikbereichen wie der Fiskalpolitik auf europäischer Ebene zu bündeln, darüber hinaus unabdingbar.

Obwohl vor dem Risiko einer Reduzierung oder Zerfaserung der Europäischen Union gewarnt wird, ${ }^{5}$ hat sich differenzierte Integration als effektives Instrument zum Management der europäischen Vielfalt durchgesetzt. ${ }^{6}$ Vor diesem Hintergrund haben sich sowohl Wissenschaftler als auch Politiker als sehr kreativ erwiesen, die verschiedensten Begriffe für dieses Managementinstrument zu prägen. Diese reichen von so bildlichen Begriffen wie einer „europäischen Zwiebel“"7 oder „fliegenden Gänsen“8 bis hin zu Konzepten der ,abgestuften

* Dr. Funda Tekin, Wissenschaftliche Mitarbeiterin am Jean Monnet Lehrstuhl Prof. Wessels, Universität zu Köln.

1 Siehe hierzu unter anderem Jean-Claude Piris: The Future of Europe. Towards a Two-Speed Europe?, Cambridge 2011; Michael Leigh: Is a Two-Speed Europe the Solution?, Washington 2012.

2 Angela Merkel im ARD-Morgenmagazin vom 7. Juni 2012. Vgl. Tagesschau.de: Merkel setzt auf politische Einheit Europas, 7.6.2012.

3 Funda Tekin/Wolfgang Wessels: Flexibility within the Lisbon Treaty: Trademark or Empty Promise?, in: Eipascope $1 / 2008$, S. 25-28, hier S. 25.

4 Anne Faber: Die Weiterentwicklung der Europäischen Union: Vertiefung versus Erweiterung, in: integration 2/2007, S. 103-116.

5 Nicolai von Ondarza: Zwischen Integrationskern und Zerfaserung. Folgen und Chancen einer Strategie differenzierter Integration, Stiftung Wissenschaft und Politik, September 2012.

6 Siehe unter anderem Kenneth Dyson/Angelos Sepos (Hrsg.): Which Europe? The Politics of Differentiated Integration, Houndmills Basingstoke 2010.

7 Jan-Emmanuel De Neve: The European Onion? How differentiated Integration is Reshaping the EU, in: Journal of European Integration 4/2007, S. 503-521.

8 Helen Wallace/William Wallace: Flying together in a larger and more diverse European Union, Scientific Council for Government Policy, Den Haag 1995. 
Integration“9 oder einem „Kerneuropa“. ${ }^{10}$ Allerdings ergibt sich aus dieser überladenen konzeptionellen Debatte das Problem, dass klare Definitionen des Begriffs , differenzierte Integration“ unter einer „Semantiklawine“11 der immer schneller anwachsenden Terminologie begraben werden. Eine Kategorisierung der differenzierten Integration rangiert daher ganz oben auf der Forschungsagenda aktueller Studien. ${ }^{12}$

\section{Abbildung 1: Europa in Vielfalt geeint}

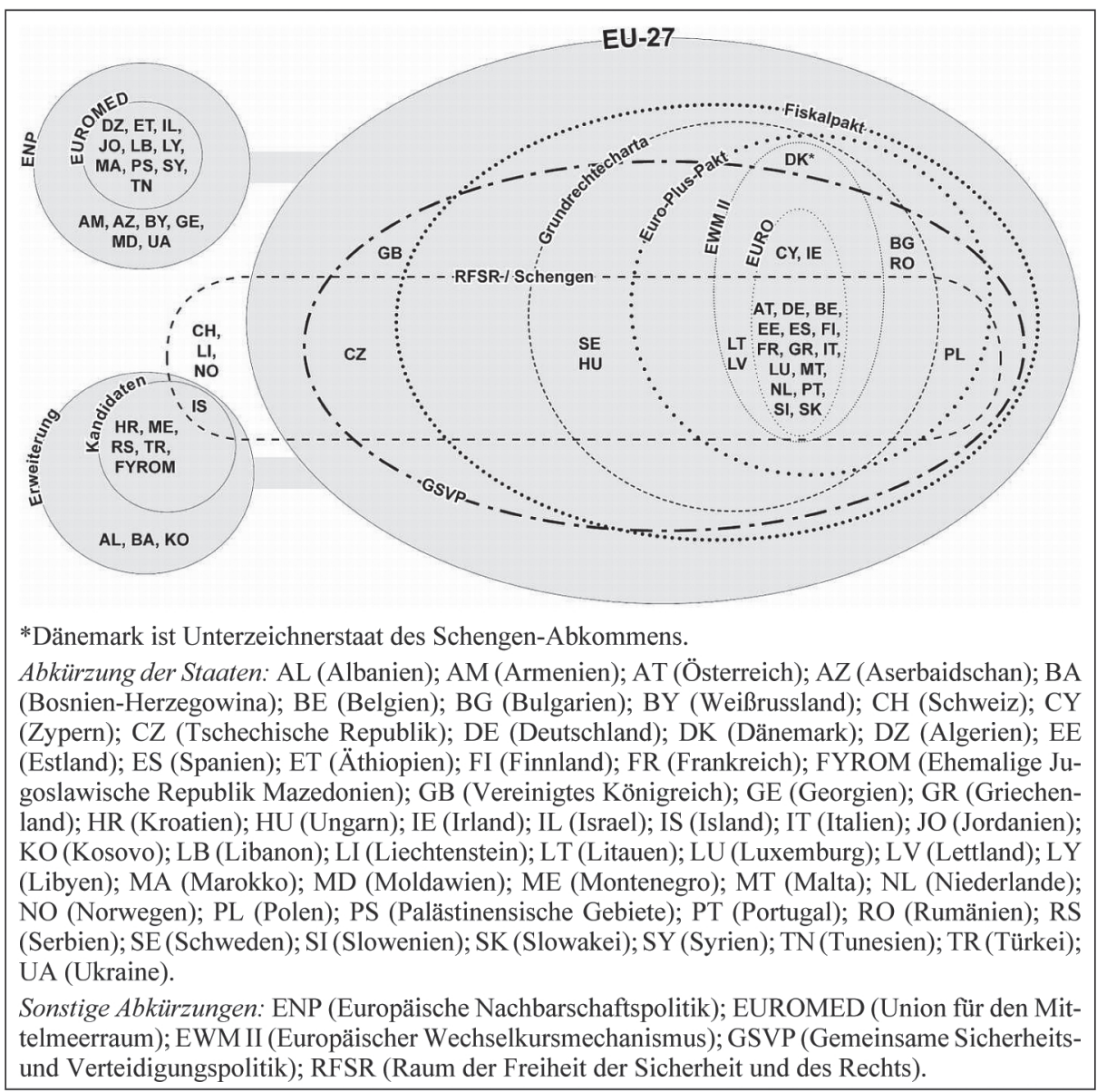

Quelle: Überarbeitete Darstellung aus Tekin: Differentiated Integration at Work, 2012, S. 20.

9 Eberhard Grabitz/Constantin Iliopoulos: Typologie der Differenzierungen und Ausnahmen im Gemeinschaftsrecht, in: Eberhard Grabitz (Hrsg.): Abgestufte Integration. Eine Alternative zum herkömmlichen Integrationskonzept?, Kehl am Rhein 1984, S. 31-44.

10 Wolfgang Schäuble/Karl Lamers: Überlegungen zur Europapolitik, in: Blätter für deutsche und internationale Politik 10/1994, S. 1271-1280.

11 Funda Tekin: Differentiated Integration at Work. The Institutionalisation and Implementation of Opt-Outs from European Integration in the Area of Freedom, Security and Justice, Baden-Baden 2012, S. 39.

12 Katharina Holzinger/Frank Schimmelfennig: Differentiated integration in the European Union: Many concepts, sparse theory, few data, in: Journal of European Public Policy 2/2012, S. 292-305. 
Seit den Anfängen der Europäischen Gemeinschaft ist differenzierte Integration in jedem Fall eine Realität der europäischen Integration. ${ }^{13}$ Selbst wenn man von den Formen abstrahiert, bei denen die Differenzierung durch unterschiedliche Rechte und Pflichten im Sekundärrecht - wie zum Beispiel bei Übergangsregelungen für neue Mitgliedstaaten oder sogenannten Sicherheitsklauseln ${ }^{14}$ - oder durch grenzüberschreitende Zusammenarbeit - wie im Europäischen Wirtschaftsraum und in der Zollunion - entsteht, ist das Bild eines ,Europas in Vielfalt geeint' höchst komplex (siehe Abbildung 1).

Insgesamt haben sieben Mitgliedstaaten in vier Politikfeldern sogenannte Opt-Out-Regeln verhandelt, die im Primärrecht der Europäischen Union verankert worden sind (siehe Tabelle 1). Eine solche Differenzierungsrealität fordert eine strukturierte Analyse, die über die konzeptionelle Debatte hinausgeht. Erstaunlicherweise wurde gemäß dem aktuellen Forschungsstand die Implementierung verschiedener Formen der differenzierten Integration und deren Konsequenzen für den europäischen Integrationsprozess bisher recht stiefmütterlich behandelt. Einzig ausgesuchte Studien unter anderem zur „Diplomatie der Opt-outs“, ${ }^{15}$ zum „heimlichtuerischen Kreis der Finanzminister der Eurogruppe“16 oder zur Europäischen Union als „System differenzierter Integration“ ${ }^{17}$ haben sich diesem neuen Forschungsfeld zugewandt.

Dieser Artikel soll einen weiteren Beitrag zu diesem Forschungstrend leisten. ${ }^{18}$ Ziel ist es, ein umfassendes und detailliertes Bild der komplexen Differenzierungsmuster im Raum der Freiheit, der Sicherheit und des Rechts (RFSR) zu zeichnen, die aus dem Zusammenspiel der geschriebenen und gelebten Vertragspraxis entstehen. Die Differenzierung in diesem Politikbereich stellt aufgrund eines hohen Grads an Differenzierungsvolatilität und sehr komplexer Opt-Out-Strukturen mit umfassenden Ad-hoc-Beteiligungsrechten für die drei Opt-Out-Staaten (siehe Tabelle 1) ein interessantes Forschungsfeld dar. Dem Artikel liegt somit die Forschungsfrage nach der Differenzierungsrealität zugrunde, die im RFSR durch die Implementierung institutionalisierter Gesetzgebungsstrukturen durch rational handelnde Akteure generiert wird. Darüber hinaus soll die Frage nach dem Nutzen der Ausnahmeregelungen für Großbritannien, Irland und Dänemark erörtert werden.

In einem ersten Schritt wird ein Ansatz zur Strukturierung der komplexen Differenzierungsrealität in der Europäischen Union vorgestellt. Daraufhin werden die Rahmenbedingungen und Grundprinzipien der Opt-Outs im RFSR herausgearbeitet. In einem letzten Schritt werden dann die Differenzierungsmuster im RFSR identifiziert und eingeordnet.

13 Frank Schimmelfennig/Dirk Leuffen/Berthold Rittberger: Ever looser union? Towards a theory on differentiated integration in the EU, 12. International Biennial Conference of the European Union Studies Association, Boston 2011.

14 Sven Andersen/Nick Sitter: Differentiated Integration: What is it and how much can the EU accommodate?, in: Journal of Common Market Studies 4/2006, S. 313-330.

15 Rebecca Adler-Nissen: The Diplomacy of Opting Out: A Bourdieudian Approach to National Integration Strategies, in: Journal of Common Market Studies 3/2008, S. 663-684.

16 Uwe Pütter: The Eurogroup. How a secretive circle of finance ministers shape the European economic governance, European Policy Research Unit Series, Manchester 2006.

17 Dirk Leuffen/Berthold Rittberger/Frank Schimmelfennig: Differentiated Integration. Explaining Variation in the European Union, Houndmills Basingstoke 2012.

18 Dieser Artikel basiert auf der Promotionsforschung der Autorin. Vgl. Tekin: Differentiated Integration at Work, 2012. 
Tabelle 1: Opt-Outs im aktuellen Vertragsrecht der Europäischen Union

\begin{tabular}{|c|c|c|c|c|c|c|c|}
\hline & & & & & Pol & kfeld & \\
\hline & & $\begin{array}{l}\text { Wirt } \\
\text { Wäh }\end{array}$ & $\begin{array}{l}\text { hafts } \\
\text { ungst }\end{array}$ & $\begin{array}{l}\text { - und } \\
\text { nion }\end{array}$ & RFSR & Verteidigung & $\begin{array}{l}\text { Grundrechte- } \\
\text { charta* }\end{array}$ \\
\hline & & $€$ & FP & EPP & & & \\
\hline & Dänemark & $\mathrm{X}$ & & & $\mathrm{X}$ & $\mathrm{X}$ & \\
\hline & Großbritannien & X & $\mathrm{X}$ & $X$ & X & & $\mathrm{X}$ \\
\hline$\frac{\overline{0}}{\pi}$ & Irland & & & & $\mathrm{X}$ & & \\
\hline$\frac{\pi}{2}$ & Polen & & & & & & $\mathrm{X}$ \\
\hline 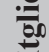 & Schweden & $\mathrm{X}^{* *}$ & & & & & \\
\hline$\sum$ & $\begin{array}{l}\text { Tschechische } \\
\text { Republik }\end{array}$ & & $\mathrm{X}$ & $X$ & & & \\
\hline & Ungarn & & $X$ & $X$ & & & \\
\hline
\end{tabular}

Quelle: Eigene Darstellung.

\section{,Differenzierte Integration bei der Arbeit': ein Strukturierungsansatz zur komplexen Differenzierungsrealität}

Im RFSR ist der Differenzierungsgrad weniger eindeutig zu bestimmen als in den anderen drei Politikbereichen, die Gegenstand von Opt-Out-Regelungen sind. Grundsätzlich sind Großbritannien und Irland kein Teil des RFSR und des Schengenraums. Aufgrund komplizierter Regeln haben diese zwei Opt-Out-Staaten jedoch umfassende Rechte, sich ad hoc zu entscheiden, an ausgesuchten Maßnahmen in diesem Bereich teilzunehmen. Auch Dänemark, welches zu den Unterzeichnerstaaten des Schengener Abkommens zählt, wurde ein umfassendes Opt-Out-Recht gewährt. Der Differenzierungsgrad in diesem Politikfeld wird demnach durch die Volatilität der tatsächlichen Teilnahme der Opt-Out-Staaten definiert.

Dies begründet die Annahme, dass die Implementierung der rechtlichen Differenzierungsregeln im RFSR eine Reihe von verschiedenen Mustern generiert. Hierbei spielen die gegenseitigen Effekte der strukturellen und funktionellen Dimension, das heißt die institutionellen Rahmenbedingungen des politischen Entscheidungsprozesses und des Gesetzgebungsverfahrens sowie die Inhalte der ,policies', eine entscheidende Rolle. Je freier Großbritannien, Irland und Dänemark auf struktureller und funktioneller Ebene sind, über ihre tatsächliche Teilnahme in bestimmten Maßnahmen des RFSR ad hoc zu entscheiden, desto größer ist die Volatilität der Differenzierung.

Das Forschungsinteresse gilt in diesem Sinne der Frage, ob die Opt-Out-Staaten tatsächlich in der Lage sind, vom „Besten aus beiden Welten“, ${ }^{19}$ nämlich außerhalb und innerhalb

19 Andrew Geddes: Getting the best of both worlds? Britain, the EU and migration policy, in: International Affairs 4/2005, S. 723-740, hier S. 723. 
des RFSR, zu profitieren. Oder ob sich aus den komplexen Strukturen der Opt-Outs und OptIns nicht doch ein Opt-Around ergibt, welches aufgrund unvorhersehbarer Konsequenzen keinen größeren Nutzen oder sogar Nachteile für die Opt-Out-Staaten bringt. Der Analyserahmen des „Pfads der differenzierten Integration bei der Arbeit“ ${ }^{\text {"20 }}$ kombiniert die Annahmen des historischen Institutionalismus ${ }^{21}$ mit der Logik der differenzierten Integration $^{22}$ für die Analyse der strukturellen und funktionellen Volatilität. Gemäß den Annahmen des historischen Institutionalismus ist zu erwarten, dass differenzierte Institutionen zäh sind und somit fortbestehen. ${ }^{23}$ Allerdings werden die Differenzierungsmuster im RFSR maßgeblich durch formelles und informelles Handeln rationaler Akteure in Reaktion auf strukturelle und funktionelle externe Effekte im RFSR determiniert. Auf der funktionalen Ebene kann der Nutzen des Opt-Outs demnach durch Opportunitätskosten des Ausschlusses aus dem entsprechenden Politikbereich sowie Spill-Over-Effekte in andere Politikbereiche, die von gemeinsamem Interesse sind, gemindert werden. Gemäß der Logik der differenzierten Integration und der Theorie des öffentlichen Gutes ${ }^{24}$ können solche negativen externen Effekte eine funktionale Anziehungskraft generieren, die auf Großbritannien, Irland oder Dänemark wirkt. Dieser Anziehungskraft kann nachgegeben werden, wenn in struktureller Hinsicht die Möglichkeit der aktiven Beteiligung im Gesetzgebungsprozess - also eine mögliche Stimmberechtigung - und damit der aktiven Mitgestaltung im RFSR existiert. Somit kann in Abhängigkeit von der institutionellen Volatilität das rationale Handeln der Akteure zu einer Reduzierung der tatsächlichen Differenzierung führen. Allerdings kann es aufgrund der Pfadabhängigkeit differenzierter Institutionen hierbei zu unintendierten Konsequenzen kommen, die die Muster der Differenzierungsrealität im RFSR beeinflussen.

\section{Differenzierungskonsequenzen eines sensiblen und stark vernetzten Politikbereichs}

Der RFSR stellt einen höchst sensiblen Politikbereich dar. Die Gewährleistung von Freiheit, innerer und äußerer Sicherheit sowie des Rechtsschutzes gehört zu den Kernelementen souveräner Nationalstaaten. Aus diesem Grund und aufgrund von unterschiedlichen nationalen Systemen für eine solche Gewährleistung ist es nicht verwunderlich, dass die europäische Zusammenarbeit in der Justiz- und Innenpolitik im Rahmen der Europäischen Gemeinschaft einen schwierigen Start hatte. Die darauf folgende Errichtung des RFSR war zudem von einer langsamen funktionellen, strukturellen und institutionellen Entwicklung geprägt. ${ }^{25}$ Anfangs, das heißt in den 1980er Jahren, war eine Zusammenarbeit im Bereich der Justiz- und Innenpolitik lediglich in intergouvernementaler, dem internationalen Recht unterliegender Form möglich. Seit der Überführung der Justiz- und Innenpolitik in den rechtlichen Rahmen der Europäischen Union mit dem Vertrag von Maastricht (1993) war dieser Politikbereich lange vom Kompromiss zwischen der intergouvernementalen dritten

20 Tekin: Differentiated Integration at Work, 2012, S. 86-92.

21 Paul Pierson: The path to European integration, in: Comparative Political Studies 2/1996, S. 123-163; Guy B. Peters: Institutional Theory in Political Science: The 'New Institutionalism', London 2005; Simon Bulmer: Politics in Time Meets the Politics of Time: Historical Institutionalism and the EU Timescape, in: Journal of European Public Policy 2/2009, S. 307-324.

22 Alkuin Kölliker: Flexibility and European Unification. The Logic of Differentiated Integration, Oxford 2006.

23 Paul Pierson: The path to European integration, in: Comparative Political Studies 2/1996, S. 123-163.

24 Mancur Olsen: Die Logik des kollektiven Handelns. Kollektivgüter und die Theorien der Gruppen, Tübingen 1968.

25 Vgl. The Federal Trust: The Institutions of JHA, Federal Trust Working Group on Justice and Home Affairs, London 2006; Jörg Monar: Justice and Home Affairs, in: Journal of Common Market Studies, Annual Review, September 2010, S. 279-292. 
Säule der polizeilichen und justiziellen Zusammenarbeit und der supranationalen Politikgestaltung in den Bereichen Visa, Asyl, Einwanderung und anderen Politiken den freien Personenverkehr betreffend, die in der EG-Säule des Unionsrechts verankert wurde, geprägt. Erst der Vertrag von Lissabon hat 2009 zumindest formal mehr strukturelle Kohärenz geschaffen, in dem er den RFSR durch die Abschaffung der Säulenstruktur vollständig vergemeinschaftet hat. Dies bedeutet, dass in allen Politiken des RFSR einschließlich der polizeilichen und justiziellen Zusammenarbeit in Strafsachen - die bisher letzte Domäne der intergouvernementalen Zusammenarbeit im RFSR - die qualifizierte Mehrheit im Rat der Europäischen Union als Abstimmungsmodalität sowie das ordentliche Gesetzgebungsverfahren anwendbar sind. Die Gesetzgebung unterliegt der Prüfung durch den Gerichtshof der Europäischen Union. Darüber hinaus obliegt der Kommission das alleinige Initiativrecht in der Gesetzgebung. Die einzige Ausnahme ist hier die polizeiliche und justizielle Zusammenarbeit in Strafsachen, wo sie sich dieses Recht mit mindestens einem Viertel der Mitgliedstaaten teilt. ${ }^{26}$

Die Sensibilität des Politikbereichs ist ebenfalls einer der Gründe warum der RFSR einen hohen Grad an Differenzierung aufweist. Darüber hinaus spielt hier die enge Verknüpfung der Einzelpolitiken eine wichtige Rolle. Die Europäische Kommission fasste dies im Jahr 1998 wie folgt zusammen: „Freiheit verliert viel von ihrer Bedeutung, wenn sie nicht in einem sicheren Umfeld und mit der vollen Unterstützung eines Rechtssystems genossen werden kann, in das alle Bürger und Gebietsansässigen der Union Vertrauen haben können“. ${ }^{27}$ Dies bedeutet, dass die für den Binnenmarkt elementare Aufhebung der inner-europäischen Grenzen für die Gewährleistung eines freien Personen-, Kapital-, Güter- und Dienstleistungsverkehrs eine engere und koordinierte Zusammenarbeit der Mitgliedstaaten in anderen elementaren Politikfeldern wie der Asylpolitik, der Kontrolle der Außengrenzen sowie der justiziellen Zusammenarbeit erfordert.

Wenn nun aber der Integrationsbedarf in den einzelnen Politikbereichen des RFSR eng miteinander verknüpft ist, so muss das Gleiche für die Differenzierungsnotwendigkeit gelten. In diesem Sinne gilt das Schengener Abkommen als Rückgrat dieser Differenzierung. Zunächst wurde die Aufhebung der gemeinsamen Grenzen und die deshalb erforderliche Kooperation, wie zum Beispiel die polizeiliche Zusammenarbeit, mit einer begrenzten Anzahl von Mitgliedstaaten auf intergouvernementaler Ebene geregelt. ${ }^{28}$ Dieses Vorgehen wird gemeinhin als die Schaffung eines ,Labors' zur Umgehung eines Integrationsstillstandes gewertet. Lediglich die zur Teilnahme bereiten und fähigen Mitgliedstaaten entscheiden sich zunächst für diese Zusammenarbeit. Solange die Laborarbeit erfolgreich verläuft ist es sehr wahrscheinlich, dass andere Mitgliedstaaten sich mittel- oder langfristig dieser anschließen werden. Darüber hinaus ist die Überführung solcher Labore in den rechtlichen Rahmen der Europäischen Union ein definierter Bestandteil der entsprechenden intergouvernementalen Verträge. Ein solches Vorgehen hat sich mittlerweile als ein erprobtes Mittel erwiesen. Im RFSR wurde es 2005 durch den Vertrag von Prüm über einen intensiveren Datenaustausch angewendet. Darüber hinaus dienten intergouvernementale Verträge außerhalb des rechtli-

26 Art. 76 AEUV.

27 Kommission der Europäischen Gemeinschaften: Mitteilung der Kommission. Auf dem Weg zu einem Raum der Freiheit, der Sicherheit und des Rechts, KOM (1998) 459, S. 1.

28 Geregelt im Schengener Durchführungsübereinkommen: Übereinkommen zur Durchführung des Übereinkommens von Schengen vom 14. Juni 1985 zwischen den Regierungen der Staaten der Benelux-Wirtschaftsunion, der Bundesrepublik Deutschland und der Französischen Republik betreffend den schrittweisen Abbau der Kontrollen an den gemeinsamen Grenzen vom 19. Juni 1990. 
chen Rahmens der Europäischen Union ebenso in der Finanz- und Schuldenkrise als willkommener Ausweg. Der Fiskalpakt ${ }^{29}$ und der Euro-Plus-Pakt ${ }^{30}$ sind aus mehreren Gründen (noch) nicht Gegenstand der EU-Verträge. Zum einen dauern Vertragsreformen der Europäischen Union sehr lange und werden aufgrund der zunehmenden Zahl der Mitgliedstaaten auch immer komplexer. Darüber hinaus war der vorsichtig geschnürte Vertrag von Lissabon gerade erst in Kraft getreten und dieses Verhandlungspaket soll und kann nicht sofort wieder aufgeschnürt werden. Die Ratifizierung der Vertragsreformen ist in einigen Mitgliedstaaten referendumspflichtig. In Zeiten der Finanz- und Schuldenkrise, welche den Euro-Skeptizismus schürt, wird eine solche Kondition den Reformprozess noch verlängern. Letztlich waren nicht alle Mitgliedstaaten bereit und/oder fähig, dem Fiskalpakt sowie dem Euro-Plus-Pakt beizutreten. Diese Vetopositionen verhinderten die Verabschiedung dieser Pakte auf der Grundlage der EU-Verträge und erforderten folglich differenzierte Integrationsformen. Auch hier wird auf eine Laborwirkung spekuliert, die mittelfristig einen Integrationsschritt innerhalb des rechtlichen Rahmens der Europäischen Union ermöglichen wird.

Indessen kann die Strategie des (vermehrten) Voranschreitens außerhalb des rechtlichen Rahmens der Europäischen Union auch Grund zur Sorge geben, da dieses das Risiko eines ,l'Europe à la carte' und somit eines Auseinanderfallens durch ein Rosinenpicken seitens der Mitgliedstaaten in sich birgt. ${ }^{31}$ Auf jeden Fall ist dieses Vorgehen keine Garantie dafür, dass letzten Endes alle Mitgliedstaaten der Europäischen Union sich diesem Integrationsschritt verpflichten. Zum Zeitpunkt der Überführung des Schengener Abkommens in den rechtlichen Rahmen der Europäischen Union waren fast alle Mitgliedstaaten der Europäischen Union bis auf Großbritannien und Irland bereit, ihre Binnengrenzen aufzuheben und hatten das Schengener Abkommen unterzeichnet. Aufgrund der Bedeutung des freien Personenverkehrs für den europäischen Binnenmarkt wurde dieser wichtige Integrationsschritt zu Lasten der Gemeinschaftsorthodoxie umgesetzt indem formale Ausnahmeregelungen festgeschrieben wurden. Angesichts der engen Politikverflechtung strahlte das Schengener Abkommen folglich nicht nur eine Integrations-, sondern auch eine Differenzierungswirkung aus. Die Gründe für diese Differenzierungsnotwendigkeit sollen im Folgenden erläutert werden.

\section{Grundprinzipien der Opt-Out-Regime im RFSR}

Weder Großbritannien, Irland noch Dänemark lehnen den RFSR als Kooperation auf europäischer Ebene in seiner Ganzheit ab. Jeder dieser Opt-Out-Staaten hat ein elementares Interesse an der Teilnahme an einzelnen Politiken oder auch Politikbereichen des RFSR. Dies stellt eine grundlegende Erklärung für die recht komplexen vertraglich festgelegten Regeln dar, die es diesen Staaten ermöglichen, sich ad hoc an den Gesetzgebungsverfahren oder Maßnahmen des RFSR zu beteiligen. Im Folgenden sollen die Gründe für die Opt-Outs von Großbritannien, Irland und Dänemark erörtert und die sich daraus ergebenden Grundprinzipien für die Opt-In-Möglichkeiten aufgezeigt werden.

29 Zum Fiskalpakt siehe auch Friedrich Heinemann/Marc-Daniel Moessinger/Steffen Osterloh: Feigenblatt oder fiskalische Zeitenwende? Zur potenziellen Wirksamkeit des Fiskalvertrags, in: integration 3/2012, S. 167-182.

30 Zum Euro-Plus-Pakt siehe auch Daniel Gros/Cinzia Alcidi: Was bringt der „Euro-plus-Pakt“?, in: integration 2/2011, S. 164-171.

31 Helmut P. Gaisbauer: Main stages of differentiated integration in JHA: A theory of clubs perspectives, 5. PanEuropean Conference on EU Politics, Portugal 2010. 


\section{Großbritannien: eine Frage der Grenzkontrollen und inneren Sicherheit}

Nicht nur seit dem Gipfeltreffen des Europäischen Rates im Dezember 2011, bei dem der britische Premierminister David Cameron in letzter Minute dem Fiskalpakt nicht zugestimmt hat, gilt Großbritannien allgemein als „schwieriger Partner“32 in der Europäischen Union. Großbritanniens Mitgliedschaft in der Europäischen Union ist von einem grundlegenden Euroskeptizismus geprägt. ${ }^{33}$ Dies bedeutet, dass die nationalstaatlichen Interessen in den Vordergrund gestellt und intergouvernementale Kooperationsformen bevorzugt werden. Im Bereich des RFSR ist die Position Großbritanniens darüber hinaus vornehmlich durch strukturelle Gegebenheiten hinsichtlich spezieller Politikbereiche begründet. Die Gewährleistung und der Schutz der inneren Sicherheit in einer schnelllebigen und zunehmend vernetzten globalen Welt stellt eine Priorität dar. Somit sind die Bekämpfung illegaler Migration, des Terrorismus sowie der grenzüberschreitenden Kriminalität Eckpfeiler der britischen Politik, die das Interesse für eine europäische Kooperation im Rahmen des RFSR begründen. Allerdings gibt es besonders in der justiziellen Zusammenarbeit systemisch begründete Grenzen einer solchen Kooperation. Großbritanniens Rechtssystem basiert, anders als in den Mitgliedstaaten des europäischen Kontinents, wo Zivilrechtssysteme vorherrschen, auf Gewohnheitsrecht. Daher ist im RFSR eine intergouvernementale Zusammenarbeit aus britischer Sicht erforderlich. ${ }^{34}$

Die britische Sonderposition im RFSR ist außerdem grundlegend durch die Inselposition Großbritanniens determiniert. Großbritannien hat keine langen durchlässigen Staatsgrenzen, sondern kann Grenzbewegungen über einige See- und Flughäfen sowie den Kanaltunnel kontrollieren. Der Schutz der inneren Sicherheit wird somit über die strikte Kontrolle der Staatsgrenzen gewährleistet, die Personenkontrollen im Land nicht zwingend erforderlich machen. Da die Aufhebung der Binnengrenzen des Schengenraums jedoch auf der Kombination von stark kontrollierten Außengrenzen und verstärkten internen Personenkontrollen basiert, ist eine Teilnahme Großbritanniens schwierig. Der britische Nutzen des grenzenlosen Verkehrs innerhalb des Schengenraums wird somit durch den Sicherheitsaspekt des Verlusts der nationalen Kontrolle über die eigenen Grenzen und die Schengen-Außengrenzen reduziert. Das Schengener Abkommen stellt in diesem Fall das Rückgrat des britischen Opt-Outs dar, welches aufgrund der oben dargelegten engen Verknüpfung der Politiken in den RFSR ausstrahlt. Großbritannien kann daher als ein ,Fremdling' im RFSR bezeichnet werden, welcher konsequent und erfolgreich seine Souveränitätsrechte wahrt und dafür auch das Risiko einer Marginalisierung in Kauf nimmt. Allerdings kann von einer großen funktionellen Anziehungskraft ausgegangen werden, weil das Interesse an den ergänzenden Politikbereichen wie Asyl- und Migrationspolitik groß ist.

\section{Irland: eine Frage der Abhängigkeit von der britischen Schengen-Teilnahme}

Irlands Agieren in der Europäischen Union ist von Pragmatismus geprägt. Irland ist sich der Tatsache bewusst, dass es ein kleiner Mitgliedstaat ist. Aus diesem Grund wird bei der Durchsetzung nationalstaatlicher Interessen darauf geachtet, Koalitionen zu bilden und,

32 Stephen George: Britain in the IGC, in: Geoffrey Edwards/Alfred Pijpers (Hrsg.): The Politics of the European Treaty Reform. The 1996 Intergovernmental Conference and Beyond, London 1997, S. 100-118, hier S. 100.

33 Antje Wiener: Forging Flexibility - the British 'No' to Schengen, Arena Working Papers Series: Working Paper $1 / 2000$.

34 Alastair Sutton: The Lisbon Treaty and the United Kingdom, Brüssel 2008, S. 9. 
wenn möglich, Konfrontationen zu vermeiden. ${ }^{35}$ Die irische Europapolitik ist somit von dem Bestreben gekennzeichnet, sich als ein ,guter Europäer“ 36 zu präsentieren. Historisch und geografisch bedingt ist Großbritannien der Hauptkoalitionspartner Irlands auf europäischer Ebene. Im RFSR ist Irland wegen struktureller Gegebenheiten dazu gezwungen, sich Großbritannien im Opt-Out anzuschließen. Diese beiden Staaten haben ein gemeinsames Reisegebiet konstituiert. Das ist besonders wichtig für die Staatsgrenze zu Nordirland, die heutzutage für Reisende und Pendler fast unsichtbar ist. Ein Beitritt Irlands zum Schengenraum würde bedeuten, dass die britisch-irische Grenze eine Schengen-Außengrenze darstellt, die verstärkt zu kontrollieren wäre. Der Reiseverkehr zwischen diesen beiden Staaten würde somit äußerst erschwert. Dies wäre besonders nachteilig da 85 Prozent des irischen Personengrenzverkehrs über die gemeinsame Grenze mit Großbritannien erfolgt. Aus diesem Grund ist es für Irland nicht von Nutzen, ein Teil des Schengenraums zu werden, solange Großbritannien nicht zum selben Schritt bereit ist. Irlands Kosten-Nutzen-Kalkül wird sozusagen durch die britische Zwangsjacke bestimmt. Diese ist besonders eng, da Irland einen Nutzen aus der Teilnahme an den anderen Politiken des RFSR ziehen könnte. Lediglich in Fragen der justiziellen Zusammenarbeit ist dieser Nutzen abhängig von der Kompatibilität der Rechtssysteme, da in Irland das Rechtssystem ebenfalls auf Gewohnheitsrecht basiert. Als ,guter Europäer' hat sich Irland jedoch verpflichtet, von den rechtlichen Opt-In-Möglichkeiten umfassend Gebrauch zu machen. ${ }^{37}$

\section{Dänemark: eine Frage des Souveränitätsverzichts}

Während die Opt-Outs von Großbritannien und Irland somit durch den grundlegenden Politikgegenstand des RFSR - der Auflösung der Binnengrenzen - bedingt sind, hat das OptOut von Dänemark seinen Ursprung nicht in einem bestimmten Politikbereich des RFSR, sondern in der Frage des nationalen Souveränitätsverzichts. Dänemark ist offiziell ein Unterzeichnerstaat des Schengener Abkommens. Ähnlich wie Irland ist Dänemark in ein gemeinsames Reisegebiet mit anderen Staaten eingebunden. Allerdings liegt der Fall hier ein wenig anders: Die Staaten der Nordischen Passunion sind alle an den Schengen-Besitzstand gebunden. Schweden und Finnland als Mitgliedstaaten der Europäischen Union stellen Schengen-Teilnehmer mit vollen Rechten dar. Norwegen und Island sind aufgrund einer fehlenden Mitgliedschaft in der Europäischen Union assoziierte Teilnehmer. Im Gegensatz zu Irland wird Dänemarks Verbleib im Schengenraum durch die Nordische Passunion erforderlich. Dass Dänemarks Versuch, 2011 Grenzkontrollen an den Grenzen zu anderen EUMitgliedstaaten wieder einzuführen, relativ schnell unterbunden wurde, liegt zum einen an den rechtlichen Verpflichtungen aus dem Schengener Abkommen und zum anderen an den Problemen, die solche Grenzkontrollen für die Nordische Passunion bedeutet hätten. Dänemark ist generell ein Befürworter von koordinierter Asyl- und Migrationspolitik im Rahmen der Europäischen Union. Dies allerdings nur unter der Prämisse, das strenge nationale Asylsystem nicht zu liberalisieren. Die justizielle Zusammenarbeit stellt, anders als bei Großbri-

35 Ben Tonra: Ireland: The Politics of Pragmatism?, in: Finn Laursen (Hrsg.): The Amsterdam Treaty: National Preference Formation, Interstate Bargaining and Outcome, Odense 2002, S. 201-224.

36 Ebenda.

37 56. Erklärung Irlands [zur Schlussakte der Regierungskonferenz, die den am 13. Dezember 2007 unterzeichneten Vertrag von Lissabon angenommen hat] zu Artikel 3 des Protokolls über die Position des Vereinigten Königreichs und Irlands hinsichtlich des Raums der Freiheit, der Sicherheit und des Rechts, in: Amtsblatt der EU, Nr. C 83 vom 30. März 2012, S. 356-357. 
tannien und Irland, aufgrund eines mehrheitlich kompatiblen Rechtssystems kein Problem dar.

Dem Opt-Out von Dänemark liegen somit keine politikinhaltlichen Faktoren, sondern die Sensibilität des Politikbereichs zugrunde. Um die nationalstaatlichen Souveränitätsrechte nicht zu gefährden, ist Dänemark lediglich bereit, im Rahmen von internationalem Recht oder intergouvernementalen Strukturen der EU-Verträge im RFSR zu partizipieren. Aufgrund dieses Schutzes der nationalen Souveränität konstituiert die dänische Verfassung ein Sicherheitsnetz für die Verabschiedung von Gesetzen, die nationale Rechte auf die supranationale Ebene transferieren. Die Referendumsnotwendigkeit sieht vor, dass ein solches Gesetz von der Bevölkerung ratifiziert werden muss, wenn es keine Fünfsechstelmehrheit im dänischen Parlament findet. ${ }^{38}$ Darüber hinaus hat Dänemark bereits im Vertrag von Maastricht festgelegt, dass seine Teilnahme auf intergouvernementale Kooperationsformen im RFSR beschränkt bleiben wird (siehe unten).

Zusammenfassend lässt sich festhalten, dass die Grundprinzipien der Ausnahmeregeln von Großbritannien, Irland und Dänemark im RFSR durch ihre individuellen rationalen Kosten-Nutzen-Kalküle hinsichtlich einer Teilnahme im RFSR einschließlich des Schengenraums determiniert sind. Großbritannien lehnt die Auflösung der europäischen Grenzen ab und ist somit auch daran gehindert, an den komplementären Politiken des RFSR teilzunehmen. Irlands Opt-Out ist aufgrund des gemeinsamen Reisegebiets mit Großbritannien ebenfalls durch den Schengen-Besitzstand bedingt. Dänemark ist in der eigentümlichen Situation, in funktionaler Hinsicht an den Schengen-Besitzstand gebunden zu sein und darüber hinaus ein inhaltliches Interesse am RFSR zu haben und gleichzeitig verpflichtet zu sein, den Status quo des Vertrags von Maastricht in struktureller Hinsicht zu wahren. Das OptOut ist also in der Kooperationsform und weniger in den Politikinhalten begründet. Diese unterschiedlichen Grundprinzipien bestimmen zum einen die Institutionalisierung der Differenzierungsstrukturen im RFSR und zum anderen die Konsequenzen ihrer Implementierung.

\section{Differenzierungsmuster der gelebten und geschriebenen Verfassung im RFSR}

Die Opt-Out-Regelungen von Großbritannien, Irland und Dänemark sind angelegt in den Verträgen der Europäischen Union, um diesen Grundprinzipien Rechnung zu tragen. Die Ausnahmeregeln gewähren zum Teil umfangreiche formale Beteiligungsrechte an den Rechtsakten aber auch in den Gesetzgebungsverfahren des RFSR. Dies ist möglich, da Großbritannien, Irland und Dänemark eine Teilnahme im RFSR nicht ganzheitlich ablehnen, sondern ein vitales Interesse vor allem an der gemeinsamen Asyl- und Migrationspolitik haben. Darüber hinaus können, basierend auf der Logik der differenzierten Integration und der Theorie der öffentlichen Güter, die Einzelpolitiken des RFSR als Klubgüter und ausschließende Netzwerkgüter definiert werden. ${ }^{39}$ Solche Güter generieren zentripetale Effekte, das heißt funktionale Anziehungskräfte, da die Opt-Out-Staaten von dem Nutzen der Politikinhalte ausgeschlossen werden können und im Zweifel darüber hinaus negativen externen Effekten ausgesetzt sind. Des Weiteren ist die Rivalität im Konsum dieser Güter neutral oder positiv, sodass die bereits teilnehmenden Mitgliedstaaten keinen Nachteil durch eine (Adhoc-)Teilnahme von Großbritannien, Irland oder Dänemark erfahren.

38 Art. 20 Grundloven (Dänische Verfassung).

39 Kölliker: Flexibility and European Unification, 2006; Gaisbauer: Main stages of differentiated integration in JHA, Portugal 2010. 
Vor diesem Hintergrund sind die vertraglich festgeschriebenen Ausnahmeregeln zu analysieren. Aufgrund der Tatsache, dass Inhalt und Umfang der Beteiligung frei gewählt werden können, ist davon auszugehen, dass die Opt-Out-Staaten in einer komfortablen Position sind, in der sie sich das Beste aus beiden Welten aussuchen können. Dies wird durch die integrative Arbeitsstruktur im Rat der Europäischen Union verstärkt, die den Vertretern Großbritanniens, Irlands und Dänemarks auf allen Ebenen das Recht einräumt, an den Verhandlungen teilzunehmen, auch wenn sie sich nicht an der Abstimmung beteiligen dürfen. Allerdings wird dieses Rosinenpicken bereits durch die Regeln zu den formalen Beteiligungsrechten eingeschränkt. Darüber hinaus kann die Nutzung der differenzierten Gesetzgebungsstrukturen durch die rational agierenden Akteure - Mitgliedstaaten sowie Kommission - zu nicht intendierten Konsequenzen führen, die den Nutzen der Ausnahmeregelungen für Großbritannien, Irland und Dänemark schmälern.

Die Analyse der geschriebenen und gelebten Verfassung unterscheidet gemäß den Protokollen der Verträge die Ausnahmeregeln, die sich auf den Schengen-Besitzstand beziehen, von den Opt-Out-/Opt-In-Regimen des restlichen Besitzstands des RFSR. Vor dem Vertrag von Lissabon beinhaltete Letzterer die Bereiche Visa, Asyl, Einwanderung und andere Politiken den freien Personenverkehr betreffend. ${ }^{40}$ Der Vertrag von Lissabon verlagerte alle Regelungen bezüglich des RFSR in den Titel V im Dritten Teil des Vertrags über die Arbeitsweise der Europäischen Union (AEUV).

\section{Institutionalisierung der geschriebenen Verfassung: Schengen-Opt-Outs und-Opt-Ins}

Die allgemeine Herausforderung für das formale Design des Opt-Out-/Opt-In-Regimes im Schengenraum ist die Etablierung der Balance zwischen eventueller ganzheitlicher oder partieller Teilnahme von Großbritannien, Irland und Dänemark sowie der Bewahrung der Kohärenz des Schengen-Besitzstandes. Somit ist die Entscheidungsfreiheit dieser drei Staaten formal limitiert.

Großbritanniens und Irlands Opt-Out ist positiv formuliert, indem das Schengen-Protokoll die anderen Staaten ermächtigt, eine verstärkte Zusammenarbeit zu gründen. Den beiden Opt-Out-Staaten wird das Recht eingeräumt, ,jederzeit [zu] beantragen, dass einzelne oder alle Bestimmungen des Schengen-Besitzstands auch auf sie Anwendung finden sollen“. ${ }^{41}$ Dabei bieten zwei grundlegende Prinzipien einem unkontrollierten Rosinenpicken seitens Großbritanniens und Irlands Einhalt.

Zum einen unterliegt das sogenannte ,Einopten“ von Großbritannien und Irland in den Schengen-Besitzstand der Zustimmung im Rat der Europäischen Union mit geltendem Einstimmigkeitserfordernis. ${ }^{42}$ Allerdings verpflichten sich die Schengenstaaten ,die größtmöglichen Anstrengungen zu unternehmen“" ${ }^{43}$ um den Opt-Out-Staaten eine umfassende Teilnahme zu ermöglichen.

Zum anderen resultiert aus der Bewahrungspflicht der Kohärenz des Schengen-Besitzstandes eine Konditionalität hinsichtlich solcher Vorschläge und Initiativen, deren Grundlage der Schengen-Besitzstand ist. Eine rechtlich formalisierte Teilnahmeverpflichtung für

40 Titel IV des Dritten Teils des Vertrag zur Gründung der Europäischen Gemeinschaft (EGV).

41 Art. 4 Protokoll Nr. 19 [zum Vertrag von Lissabon] über den in den Rahmen der Europäischen Union einbezogenen Schengen-Besitzstand, in: Amtsblatt der EU, Nr. C 83 vom 30. März 2012, S. 290-292.

42 Ebenda.

43 45. Erklärung [zum Vertrag von Amsterdam] zu Artikel 4 des Protokolls zur Einbeziehung des SchengenBesitzstands in den Rahmen der Europäischen Union, in: Amtsblatt der EU, Nr. C 340 vom 10. November 1997, S. 140-141. 
Vorschläge und Initiativen, die den Schengen-Besitzstand weiterentwickeln in den Großbritannien und Irland ,eingeoptet ${ }^{6}$ haben, existiert erst seit dem Vertrag von Lissabon. ${ }^{44}$ Vorher war dies Gegenstand einer politischen Verpflichtung. Im Umkehrschluss bedeutet dies, dass Großbritannien und Irland von Vorschlägen und Initiativen ausgeschlossen werden, deren Grundlage der Schengen-Besitzstand ist, an dem diese Staaten nicht teilnehmen. Wenn Großbritannien und Irland in eine auf Schengen-Besitzstand gründende Initiative nicht ,einopten', hat dies zur Konsequenz, dass auch der zugrundeliegende Besitzstand nicht mehr zwangsläufig auf Großbritannien und Irland Anwendung findet. ${ }^{45}$ Der Rat der Europäischen Union oder der Europäische Rat legen mit qualifizierter Mehrheit fest, ob und wenn ja in welchem Ausmaß der grundlegende Schengen-Besitzstand für Großbritannien und Irland weiterhin anwendbar ist. Falls aus dieser differenzierten Anwendbarkeit des Besitzstands Kosten entstehen, kann der Rat der Europäischen Union beschließen, dass Großbritannien und Irland hierfür finanziell aufkommen müssen. ${ }^{46}$

Die Ausnahmeregelungen für Dänemark hinsichtlich des Schengen-Besitzstands konstituieren einen kuriosen Status, der diesen Staat im RFSR immer weiter marginalisiert. Obwohl Dänemark ein Unterzeichnerstaat des Schengener Abkommens ist, wird dieser Staat von dem Teil der Schengengesetzgebung ausgeschlossen, der Gegenstand supranationaler Gesetzgebungsverfahren ist. Dänemark hat das Recht, solche Vorschläge und Initiativen bis zu sechs Monate nach deren Verabschiedung in einzelstaatliches Recht umzusetzen. ${ }^{47}$ Allerdings wird ihm das Recht verwehrt, seine Interessen im Gesetzgebungsverfahren als gleichberechtigter Verhandlungspartner einzubringen. Dänemark wird somit zum ,decision-taker ${ }^{6}$. Das bedeutet, dass Dänemark zu Gunsten der Entscheidungsfreiheit und der Wahrung der intergouvernementalen Kooperationsform auf eine Gleichberechtigung mit den anderen Schengenstaaten der Europäischen Union verzichtet. Bis zum Vertrag von Lissabon war Dänemark zumindest in der intergouvernemental strukturierten polizeilichen und justiziellen Zusammenarbeit in Strafsachen gleichberechtigt beteiligt, weil hier die nationalen Interessen durch das Vetorecht gewahrt blieben und folglich das Opt-Out nicht griff. Durch die Auflösung der dritten Säule und der Vergemeinschaftung des gesamten RFSR im Vertrag von Lissabon werden die Einflussmöglichkeiten Dänemarks im Schengenraum vollständig beschnitten. Um einer gänzlichen Marginalisierung Dänemarks hier vorzubeugen, gewährleistet das Protokoll Nr. 22 des Vertrags von Lissabon Dänemark die Möglichkeit, Beteiligungsrechte anzuwenden, die den komplexen Verfahren von Großbritannien und Irland im Titel V AEUV (siehe unten) entsprechen. Da ein solches Verfahren jedoch voraussetzt, dass hinsichtlich der entsprechenden Maßnahme die einschlägigen Bestimmungen der EU-Verträge gelten sowie der gesamte Schengen-Besitzstand für Dänemark als Unionsrecht bindend wird ${ }^{48}$ und dies den Transfer nationalstaatlicher Souveränitätsrechte an die supranationale Ebene der Europäischen Union impliziert, setzt eine solche Anwendung die Ratifizierung mittels eines Referendums in Dänemark voraus.

44 Art. 5 Protokoll Nr. 19 [zum Vertrag von Lissabon] über den in den Rahmen der Europäischen Union einbezogenen Schengen-Besitzstand.

45 Ebenda.

46 47. Erklärung [zur Schlussakte der Regierungskonferenz, die den am 13. Dezember 2007 unterzeichneten Vertrag von Lissabon angenommen hat] zu Artikel 5 Absätze 3, 4 und 5 des Protokolls über den in den Rahmen der Europäischen Union einbezogenen Schengen-Besitzstand, in: Amtsblatt der EU, Nr. C 83 vom 30. März 2012, S. 352.

47 Art. 4 Protokoll Nr. 22 [zum Vertrag von Lissabon] über die Position Dänemarks, in: Amtsblatt der EU, Nr. C 340 vom 10. November 1997, S. 299-303.

48 Art. 8 Anhang zum Protokoll Nr. 22 über die Position Dänemarks. 


\section{Institutionalisierung der geschriebenen Verfassung: Titel V AEUV Opt-Outs und Opt-Ins}

Während die Ausnahmeregelungen für Großbritannien und Irland in den Bereichen Visa, Asyl, Einwanderung und andere Politiken betreffend den freien Personenverkehr im Vergleich zu den Ausnahmeregeln im Bereich des Schengen-Besitzstands komplizierter sind, hat Dänemark gemäß den aktuell anzuwendenden Rechtsvorschriften lediglich ein Opt-Out ohne weitere Beteiligungsrechte.

Die Rechtsvorschriften legen für Großbritannien und Irland umfangreiche Beteiligungsrechte im Rahmen eines höchst komplexen Opt-In-Regimes fest. Dieses sieht die Möglichkeit vor, sowohl in einen bereits beschlossenen Rechtsakt als auch in ein laufendes Gesetzgebungsverfahren ,einzuopten'. Dies bedeutet, dass Großbritannien und Irland, als gleichwertige Mitgliedstaaten der Europäischen Union, die Politik des RFSR mitgestalten können, wenn sie sich entscheiden, aktiv am Gesetzgebungsverfahren teilzunehmen. Dadurch wird das Menü für eine Teilnahme ,à la carte‘ um die Möglichkeit der aktiven Mitgestaltung erweitert. Obwohl dieses Opt-In-Regime nicht der Einstimmigkeit im Rat der Europäischen Union (siehe oben) unterliegt, gibt es auch hier Regeln, die die Entscheidungsfreiheit von Großbritannien und Irland einschränken:

1. Die detaillierten Rechtsvorgaben definieren explizite Zeitfenster für die Möglichkeit der Teilnahme an den Gesetzgebungsverfahren. Großbritannien und Irland haben drei Monate nach der Vorlage eines Vorschlags oder einer Initiative durch die Kommission Zeit, dem Präsidenten des Rats schriftlich mitzuteilen, dass sie sich an der Annahme und Anwendung der betreffenden Maßnahme beteiligen möchten. ${ }^{49}$ Obwohl die Kommission Gesetzesvorschläge nicht unvermittelt vorlegt und diese durch den Rahmen der vom Europäischen Rat beschlossenen mehrjährigen Aktionsprogramme vorgegeben sind, erhöht die Vorgabe von drei Monaten den Zeitdruck für den nationalen Entscheidungsprozess hinsichtlich des entsprechenden Rechtsakts in Großbritannien und Irland. Wenn dieses Zeitfenster nicht eingehalten werden kann, verlieren diese zwei Opt-Out-Staaten zwar das Recht auf aktive Mitgestaltung, sie können aber dennoch an der Maßnahme teilnehmen, wenn sie nach deren Verabschiedung ,einopten' ${ }^{50}$ Hierfür gelten die Regeln der verstärkten Zusammenarbeit. ${ }^{51}$

2. Die aktive Mitgestaltung im Gesetzgebungsverfahren von Großbritannien und Irland ist darüber hinaus durch das Verbot einer Blockadehaltung limitiert. Dies bedeutet, dass die Volatilität der Differenzierung hier in beide Richtungen funktioniert. Großbritannien und Irland können von einem Gesetzgebungsverfahren ausgeschlossen werden, wenn aufgrund ihrer Verhandlungspositionen die Maßnahme nicht innerhalb eines angemessenen Zeitraums angenommen werden kann. ${ }^{52}$

3. Auch hinsichtlich des Titels IV EGV beziehungsweise des Titels V AEUV gilt das Kohärenz- und Operabilitätskriterium. Der Rat der Europäischen Union hat in diesem Verfahren das Recht, Großbritannien und Irland aufzufordern, an einer Maßnahme teilzunehmen, die den Besitzstand weiterentwickelt von dem diese Opt-Out-Staaten ein Be-

49 Art. 3 Protokoll Nr. 21 [zum Vertrag von Lissabon] über die Position des Vereinigten Königreichs und Irlands hinsichtlich des Raums der Freiheit, der Sicherheit und des Rechts, in: Amtsblatt der EU, Nr. C 83 vom 30. März 2010, S. 295-298.

50 Art. 4 Protokoll Nr. 21 [zum Vertrag von Lissabon] über die Position des Vereinigten Königreichs und Irlands.

51 Art. 331 Abs. 1 AEUV.

52 Art. 3 Abs. 2 Protokoll Nr. 21 [zum Vertrag von Lissabon] über die Position des Vereinigten Königreichs und Irlands. 
standteil sind. ${ }^{53}$ Wenn Großbritannien und Irland dennoch davon absehen, eine entsprechende schriftliche Mitteilung an den Rat der Europäischen Union zu richten, haben sie wiederum die Möglichkeit, nach der Verabschiedung des relevanten Rechtsaktes ,einzuopten'. Falls sie sich auch dagegen entscheiden sollten, wird der Rechtsakt in den beiden Staaten keine Anwendung finden. Dies hat zur Konsequenz, dass die ursprüngliche Maßnahme, die diesen Rechtsakt begründet, ebenfalls nicht mehr auf Großbritannien und Irland anwendbar ist. Der Rat der Europäischen Union kann darüber hinaus mit qualifizierter Mehrheit beschließen, dass Großbritannien und Irland die finanziellen Konsequenzen einer solchen Situation zu tragen haben. Das Maßregelungspotenzial einer solchen Regelung wird in Brüssel jedoch gemeinhin als ,zahnloser Tiger ' interpretiert. Denn die finanziellen Konsequenzen einer differenzierten Anwendbarkeit des Besitzstandes werden schwer ermittelbar sein. Daher besteht hier die Möglichkeit für Großbritannien und Irland sich unliebsamer Rechtsakte, die im Zeitverlauf unintendierte Konsequenzen generiert haben, zu entledigen.

Das Opt-Out Dänemarks hat seine Basis im sogenannten Edinburgh Abkommen von 1992. In diesem legte der Europäische Rat fest, dass „,Dänemark in den Bereichen Justiz und Inneres auf der Grundlage des Titels VI des Vertrags über die Europäische Union [(EUV) in der Fassung des Vertrags von Maastricht] uneingeschränkt teilnehmen [wird] “. 54 Da zu diesem Zeitpunkt die Justiz- und Innenpolitik der Europäischen Union gänzlich in der dritten Säule, das heißt in Titel VI EUV in der Fassung des Vertrags von Maastricht, geregelt war, handelte es sich um eine Präventivmaßnahme. Diese sollte jedoch mit jeder Vertragsreform und folglich mit der stufenweisen Vergemeinschaftung des RFSR weitreichende Konsequenzen zeigen. Das Protokoll Nr. 22 über die Position Dänemarks stipuliert ein klar umrissenes OptOut, indem Art. 1 eine dänische Beteiligung an der Annahme von Maßnahmen durch den Rat, die nach dem Titel V AEUV vorgeschlagen werden, ausschließt. Darüber hinaus stellt Art. 2 fest, dass sowohl die Rechtsakte des Titel V AEUV als auch die entsprechende Rechtsprechung des Gerichtshofs der Europäischen Union für Dänemark nicht bindend sind. Da im AEUV der gesamte RFSR einschließlich der polizeilichen und justiziellen Zusammenarbeit in Strafsachen im Titel V AEUV geregelt ist, wird Dänemark mit dem Grad der ,Lissabonisierung' des RFSR-Besitzstands marginalisiert und letztendlich aus dem RFSR ausgeschlossen. Um dem vorzubeugen, ist es möglich, dass Dänemark hinsichtlich spezieller Maßnahmen mit den anderen 26 Mitgliedstaaten der Europäischen Union sogenannte Parallelabkommen abschließt, die Gegenstand des internationalen Rechts sind. Da diese bilateralen Abkommen jedoch als artifizielles Mittel angesehen werden, sieht das Protokoll Nr. 22 zum Vertrag von Lissabon auch für den Besitzstand des Titel V AEUV die Möglichkeit vor, dass Dänemark entsprechend seiner verfassungsrechtlichen Vorschriften mitteilen kann, dass es das Opt-In-Regime anwendet, welches im Anhang dieses Protokolls geregelt wird. Wie oben beschrieben, handelt es sich hierbei um dieselben Regeln, die bereits aktuell für Großbritannien und Irland im Titel V AEUV gelten.

53 Art. 4a Abs. 2 Protokoll Nr. 21 [zum Vertrag von Lissabon] über die Position des Vereinigten Königreichs und Irlands.

54 Dänemark und der Vertrag über die Europäische Union, Beschluss der im Europäischen Rat vereinigten Staatsund Regierungschefs zu bestimmten von Dänemark aufgeworfenen Problemen betreffend den Vertrag über die Europäische Union, Edinburgh 11./12.12.1992, in: Amtsblatt der EU, Nr. C 348 vom 31. Dezember 1992, S. 1-4, hier Abschnitt D. 


\section{Implementationsformen: Opt-Arounds in Schengen?}

Der Implementierungsspielraum der formalen Differenzierungsstrukturen hinsichtlich des Schengen-Besitzstands ist relativ gering. In jedem Fall müssen Großbritannien, Irland und Dänemark den fertigen Rechtsakt akzeptieren, weil sie hier kein Recht auf aktive Mitgestaltung im Gesetzgebungsprozess eingeräumt bekommen. Die Konditionalität der Zustimmung durch den Rat der Europäischen Union sowie der ganzheitlichen Teilnahme an einem speziellen Teil des Besitzstands führt zu einer recht rigiden Volatilität. Dies bedeutet, dass die Opt-Out-Staaten langfristig entweder dabei oder nicht dabei sind. Weder Großbritannien noch Irland mussten bisher ermahnt werden, ihrer Teilnahmeverpflichtung in einer Maßnahme nachzukommen. Dagegen wurde diesen beiden Staaten bereits in drei Fällen der Zugang zu Rechtsakten verwehrt, weil sie kein Teil des zugrunde liegenden Schengen-Besitzstands waren. Hierbei handelt es sich um die Verordnung über Normen für Sicherheitsmerkmale und biometrische Daten in von den Mitgliedstaaten ausgestellten Pässen und Reisedokumenten, ${ }^{55}$ die Verordnung zur Errichtung von FRONTEX ${ }^{56}$ sowie den Beschluss über den Zugang der Mitgliedstaaten und von Europol zum Visa-Informationssystem. ${ }^{57}$ Dieser Ausschluss wurde nach britischer Klage durch den Gerichtshof der Europäischen Union bestätigt. ${ }^{58}$ Die Urteile haben die Rigidität der institutionalisierten Differenzierung hinsichtlich des Schengen-Besitzstandes untermauert. Das dient zum einen der Verhinderung eines Rosinenpickens seitens Großbritanniens und Irlands. Zum anderen können durch die eingeschränkte Entscheidungsfreiheit hinsichtlich spezieller Schengen-Maßnahmen durch solche Zwangs-Opt-Ins oder verwehrten Opt-Ins die strukturell bedingten Kosten des allgemeinen Schengen-Opt-Outs dieser beider Staaten soweit in die Höhe getrieben werden, dass deren Kosten-Nutzen-Kalkül sich langfristig zugunsten der Aufhebung dieser Ausnahmeregelung verschieben könnte.

Da Dänemark das Schengener Abkommen unterzeichnet hat und lediglich das Recht eingeräumt bekommen hat, den Schengen-Besitzstand als Gegenstand des internationalen Rechts anzuwenden, ist die funktionelle Volatilität sehr gering bis gar nicht existent. Die dänische Teilnahme an den Vorschlägen und Initiativen, deren Grundlage der SchengenBesitzstand ist, gilt nach deren Verabschiedung als vorausgesetzt. Aufgrund dieser obligatorischen Teilnahme ist die Entscheidungsfreiheit von Dänemark sehr limitiert. Folglich implementiert Dänemark 100 Prozent des Schengen-Besitzstandes unter zusätzlichen Implementierungskosten und ohne das Recht zu haben, offiziell Einfluss auf diese Rechtsakte nehmen zu können. Die Referendumspflicht zur Änderung des Opt-In-Regimes erschwert die Erhöhung der Volatilität. Ein solches Referendum wird auch die anderen Opt-Outs Dänemarks (siehe Tabelle 1) umfassen. Trotz wiederholter Ankündigung wird das Referendum aufgrund der Euro-Krise immer wieder verschoben.

55 Verordnung (EG) Nr. 2252/2004 des Rates vom 13. Dezember 2004 über Normen für Sicherheitsmerkmale und biometrische Daten in von den Mitgliedstaaten ausgestellten Pässen und Reisedokumenten, in: Amtsblatt der EU, Nr. L 385 vom 29. Dezember 2004, S. 1-6.

56 Verordnung (EG) Nr. 2007/2004 des Rates vom 26. Oktober 2004 zur Errichtung einer Europäischen Agentur für die operative Zusammenarbeit an den Außengrenzen der Mitgliedstaaten der Europäischen Union, in: Amtsblatt der EU, Nr. L 349 vom 25. November 2004, S. 1-11.

57 Beschluss 2008/633/JI des Rates vom 23. Juni 2008 über den Zugang der benannten Behörden der Mitgliedstaaten und von Europol zum Visa-Informationssystem (VIS) für Datenabfragen zum Zwecke der Verhütung, Aufdeckung und Ermittlung terroristischer und sonstiger schwerwiegender Straftaten, in: Amtsblatt der EU, Nr. L 218 vom 13. August 2008, S. 129-136.

58 Für eine genaue Analyse siehe Maria Fletcher: Schengen, the European Court of Justice and Flexibility under the Lisbon Treaty: Balancing the United Kingdom's 'Ins' and 'Outs', in: European Constitutional Law Review 1/2008, S. 71-98. 


\section{Implementationsformen: Opt-Arounds in Title V AEUV?}

Die funktionelle und strukturelle Volatilität der Ausnahmeregelungen hinsichtlich des Titels IV EGV beziehungsweise des Titels V AEUV ist höher als im Schengenraum. Während Großbritannien und Irland die Politikinhalte frei wählen können und darüber hinaus auch aktiv am Gesetzgebungsprozess teilnehmen dürfen, sieht sich Dänemark mit einem drohenden Ausschluss aus dem RFSR aufgrund der Vergemeinschaftung des Besitzstandes und der Gesetzgebung konfrontiert. Vier Muster der Differenzierungsrealität können hinsichtlich der Implementierung der komplexen Opt-Out-/Opt-In-Regime identifiziert werden:

Erstens, für die Teilnahme an den Initiativen, die bestehendes Recht weiterentwickeln, haben Großbritannien, Irland und Dänemark weder einen Freifahrtsschein noch eine Teilnahmegarantie.

Sowohl ein Zwangs-Opt-In als auch ein Opt-Out-Rauswurf, die rein formal möglich wären, haben so noch nicht stattgefunden. Da der Aufbau eines politischen Zwangs zur Teilnahme an einer Initiative schwierig ist, bedient sich die Kommission eher rechtlicher Mittel. Der Vorschlag zur Änderung der Verordnung zur Festlegung von Normen für die Aufnahme von Asylbewerbern stellt zum Beispiel eine Neufassung dar. ${ }^{59}$ Diese legt fest, dass die ursprüngliche Verordnung durch die Neufassung ersetzt wird. Folglich verliert die ursprüngliche Verordnung, die bindend für Großbritannien und Irland ist, ihre Existenz. Um weiterhin ein Teil der Verordnung zur Festlegung von Normen für die Aufnahme von Asylbewerbern sein zu können, müssten Großbritannien und Irland daher in die Neufassung, einopten`. Weder Irland noch Großbritannien haben dies bis zum heutigen Zeitpunkt getan. Irland verzichtet regelmäßig auf die aktive Teilnahme am Gesetzgebungsprozess, weil das Zeitfenster der drei Monate nicht eingehalten werden kann. Daher ist davon auszugehen, dass Irland nach der Verabschiedung der Neufassung der Verordnung dieser beitreten wird. Der Fall von Großbritannien stellt sich indessen ein wenig anders dar, weil Großbritannien trotz eines bereits existierenden hohen Standards der nationalen Normen für die Aufnahme von Asylbewerbern sich hierzu nicht auf europäischer Ebene verpflichten möchte. Um Großbritannien jedoch zumindest auf die ursprüngliche Verordnung zu verpflichten, hat der Rat der Europäischen Union die Regelung in der Neufassung dahingehend interpretiert, dass die Auflösung der ursprünglichen Verordnung durch die Neufassung für Großbritannien nicht gilt, weil die Neufassung auf Großbritannien keine Anwendung findet. Somit wird das Opt-In Großbritanniens auf dem Stand der ursprünglichen Verordnung sozusagen ,eingefroren'.

Bis zum ersten Halbjahr 2010 waren aus politischen Gründen weder Großbritannien noch Irland von einem Gesetzgebungsverfahren, in welches sie ,eingeopted“ hatten, wieder ausgeschlossen worden, obwohl innerhalb eines angemessenen Zeitraums keine Entscheidungsfindung möglich war. ${ }^{60}$ Ein solcher Opt-In-Rauswurf hat nun jedoch seinen Präzedenzfall in der Europäischen Schutzanordnung ${ }^{61}$ gefunden. Großbritannien hatte in das Gesetzgebungsverfahren ,eingeoptet', um Einfluss auf die Rechtsgrundlage der Europäischen Schutzanordnung nehmen zu können. Der Vorschlag für diese Richtlinie verankerte die Europäische Schutzanordnung im Kriminalrecht, welches nicht kompatibel mit dem britischen Rechts-

59 Kommission der Europäischen Union: Geänderter Vorschlag für eine Richtlinie des Europäischen Parlaments und des Rates zur Festlegung von Normen für die Aufnahme von Asylbewerbern (Neufassung), KOM (2011) 320.

60 Eine solche Ausschlussmöglichkeit ist vorgesehen in Art. 3 Abs. 2 Protokoll Nr. 21 [zum Vertrag von Lissabon] über die Position des Vereinigten Königreichs und Irlands.

61 Richtlinie 2011/99/EU des Europäischen Parlaments und des Rates vom 13. Dezember 2011 über die Europäische Schutzanordnung, in: Amtsblatt der EU, Nr. L 338 vom 21. Dezember 2011, S. 2-18. 
system war. Folglich war Großbritannien bereit, im Rat der Europäischen Union gegen diese Richtlinie zu stimmen und stellte dabei das Zünglein an der Waage für ein mögliches Scheitern der qualifizierten Mehrheitsentscheidung dar. Die Europäische Schutzanordnung war jedoch eine der Prioritäten der spanischen Ratspräsidentschaft 2010. Diese beschloss, Großbritannien von der Abstimmung auszuschließen, damit die Richtlinie verabschiedet werden konnte. Solch ein Ausschluss stellt eine nicht intendierte Konsequenz dar, die den Nutzen der Opt-In-Regime für Großbritannien und Irland schmälert.

Obwohl Dänemarks Teilnahmerechte am RFSR-Besitzstand bereits stark limitiert sind, erhöhen sich die Kosten des Opt-Outs darüber hinaus noch um die Möglichkeit einer OptIn-Verwehrung. Da die bilateralen sogenannten Parallelabkommen zwischen Dänemark und den restlichen Mitgliedstaaten der Europäischen Union als artifiziell gelten, kommen diese nicht zwangsläufig zustande. Grundsätzlich gilt, dass Parallelabkommen bei Initiativen notwendig sind, die ein Teil eines Systems gegenseitiger Verantwortlichkeiten sind, wie zum Beispiel in der justiziellen Zusammenarbeit in Zivilfragen. Ein Ausschluss Dänemarks wäre weder für Dänemark noch die anderen Mitgliedstaaten von Nutzen. Parallelabkommen zu einzelnen, für sich stehenden Initiativen, wie zum Beispiel die Verordnung zu Insolvenzverfahren, ${ }^{62}$ wurden jedoch bereits mehrmals seitens der Europäischen Union abgelehnt. Generell gilt, dass die spezielle Sonderposition von Dänemark allgemein zu unterbinden sei und daher Parallelabkommen zukünftig nur in Ausnahmefällen zustande kommen sollen.

Zweitens kann es zu Spill-Over-Effekten der Opt-Outs und Opt-Ins zwischen Initiativen aufgrund von Querverweisen kommen. Die Entscheidung für ein Opt-Out oder ein Opt-In hinsichtlich einer speziellen Initiative kann wegen der engen Politikverflechtung im RFSR ein Opt-Out beziehungsweise ein Opt-In in anderen Initiativen notwendig machen. Ein solcher Opt-Out-/Opt-In-Spill-Over ist in Querverweisen zwischen den Initiativen begründet. Diese tauchen insbesondere im Zusammenhang mit der Etablierung des Gemeinsamen Europäischen Asylsystems auf. So beinhaltet die Dublin-II-Verordnung ${ }^{63}$ Querverweise zur Neufassung der Verordnung zur Festlegung von Normen für die Aufnahme von Asylbewerbern ${ }^{64}$ sowie der Neufassung von EURODAC. ${ }^{65}$ Da Großbritannien nicht bereit ist, sich der Neufassung der Verordnung zur Festlegung von Normen für die Aufnahme von Asylbewer-

62 Verordnung (EG) Nr. 1346/2000 des Rates vom 29. Mai 2000 über Insolvenzverfahren, in: Amtsblatt der EU, Nr. L 160 vom 30. Juni 2000, S. 1-18.

63 Verordnung (EG) Nr. 343/2003 des Rates vom 18. Februar 2003 zur Festlegung der Kriterien und Verfahren zur Bestimmung des Mitgliedstaats, der für die Prüfung eines von einem Drittstaatsangehörigen in einem Mitgliedstaat gestellten Asylantrags zuständig ist, in: Amtsblatt der EU, Nr. L 50 vom 25. Februar 2003, S. 1-10.

64 Der Kommissionsvorschlag zur Neufassung der Dublin-II-Verordnung von 2008 definiert den allgemeinen Zusammenhang dieser drei Rechtsvorschriften auf diesem Gebiet. Darüber hinaus enthalten einzelne Artikel ausdrückliche Verweise auf die anderen Rechtsakte: zum Beipiel Art. 6 ,Garantien für Minderjährige', Art. 27 ,Gewahrsam“ und Art. 30 ,Austausch relevanter Informationen vor der Überstellung‘. Vgl. Kommission der Europäischen Gemeinschaften: Vorschlag für eine Verordnung des Europäischen Parlaments und des Rates zur Festlegung der Kriterien und Verfahren zur Bestimmung des Mitgliedstaats, der für die Prüfung eines von einem Drittstaatsangehörigen oder Staatenlosen in einem Mitgliedstaat gestellten Antrags auf internationalen Schutz zuständig ist, KOM (2008) 820.

65 Europäische Kommission: Geänderter Vorschlag Verordnung des Europäischen Parlaments und des Rates über die Einrichtung von „EURODAC“ für den Abgleich von Fingerabdruckdaten zum Zwecke der effektiven Anwendung der Verordnung (EU) Nr. [.../...] (zur Festlegung der Kriterien und Verfahren zur Bestimmung des Mitgliedstaats, der für die Prüfung eines von einem Drittstaatsangehörigen oder Staatenlosen in einem Mitgliedstaat gestellten Antrags auf internationalen Schutz zuständig ist) und für der Strafverfolgung dienende Anträge der Strafverfolgungsbehörden der Mitgliedstaaten und Europols auf den Abgleich mit EURODACDaten sowie zur Änderung der Verordnung (EU) Nr. 1077/2011 zur Errichtung einer Europäischen Agentur für das Betriebsmanagement von IT-Großsystemen im Raum der Freiheit, der Sicherheit und des Rechts, KOM (2012) 254. 
bern zu verpflichten, Rechtsakte aber in ihrer Ganzheit Anwendung finden, droht dieses OptOut auf die Verordnungen EURODAC und Dublin-II überzuschwappen. Großbritannien nimmt jedoch am Gesetzgebungsverfahren zur Revision der Dublin-II-Verordnung teil und kann somit seinen Einfluss, von Eigeninteressen geleitet, geltend machen.

Drittens können die formalen Beteiligungsrechte hinsichtlich der aktiven Mitgestaltung im Gesetzgebungsverfahren sowohl von den Opt-Out-Staaten als auch von den restlichen Staaten missbraucht werden. Großbritannien war es wiederholt möglich, den Inhalt von Initiativen gemäß seinen Interessen zu verändern, obwohl es keine offizielle Mitteilung für die Beteiligung am Gesetzgebungsverfahren eingereicht hatte. Ein solcher Missbrauch des OptIn-Regimes kann so aussehen, dass Großbritannien bei sehr technischen Verordnungen Details im Gesetzestext fordert, die es diesem Staat nach der Verabschiedung der Verordnung ermöglichen, diese anzuwenden. Solange ein solches Versprechen auf eine spätere Teilnahme Großbritanniens eingehalten wird, ist das Ausmaß des Missbrauchs relativ gering. Der Fall liegt anders, wenn dieses Versprechen nicht gehalten wird. So sorgte die Ablehnung Großbritanniens gegenüber der Neufassung der Verordnung zur Festlegung von Normen für die Aufnahme von Asylbewerbern für Irritation. ${ }^{66}$ Als besonders speziell gilt der Fall der Verordnung zum sogenannten Familien-Verfahren. Hier war es Großbritannien, trotz fehlender offizieller Teilnahme am Gesetzgebungsverfahren, nicht nur möglich, bestimmte Bedingungen an ein späteres ,Einopten` zu stellen, sondern darüber hinaus gewährt diese Verordnung weitere Ausnahmeregelungen für Großbritannien. ${ }^{67}$ Irland ist aufgrund der Tatsache, dass es einen kleinen Mitgliedstaat darstellt, in einer schwächeren Position, die formalen Beteiligungsrechte missbräuchlich zu nutzen. Des Weiteren tendiert Irland generell dazu, sich das Resultat der Gesetzgebung anzuschauen, um dann über eine Anwendung in Irland zu entscheiden.

Die missbräuchliche Auslegung der formalen Beteiligungsrechte für die Opt-Out-Staaten ist jedoch auch durch die restlichen Mitgliedstaaten der Europäischen Union möglich. Besonders im Zusammenhang mit bilateralen Abkommen mit Drittstaaten wie zum Beispiel dem SWIFT-Abkommen ${ }^{68}$ kann es passieren, dass das Verfahren abgeschlossen wird bevor das dreimonatige Zeitfenster für die Mitwirkungsbenachrichtigung seitens Irland und Großbritannien geschlossen ist.

Viertens werden in einigen Fällen maßgeschneiderte Opt-In-Lösungen erforderlich. Aufgrund der ,Initiative für eine bessere Gesetzgebung ' werden einige Rechtsakte zusammen kodifiziert. Da Großbritannien und Irland nicht zwangsläufig an allen zu kombinierenden Rechtsakten im gleichen Maße beteiligt sind, erfordert eine solche Kodifizierung maßgeschneiderte Opt-In-Regelungen. Das prominenteste Beispiel hierfür ist das Legislativpaket zur Errichtung einer Agentur für das Betriebsmanagement von IT-Großsystemen im Bereich

66 Die ursprüngliche Fassung dieses Rechtsaktes war speziell auf die britischen Konditionen zugeschnitten worden. Durch ein Opt-Out Großbritanniens in Bezug auf die Neufassung des Rechtsakts scheinen diese Konzessionen seitens der anderen Mitgliedstaaten überflüssig gewesen zu sein, weil der Rechtsakt keine Anwendung in Großbritannien mehr finden wird.

67 Verordnung (EG) Nr. 4/2009 des Rates vom 18. Dezember 2008 über die Zuständigkeit, das anwendbare Recht, die Anerkennung und Vollstreckung von Entscheidungen und die Zusammenarbeit in Unterhaltssachen, in: Amtsblatt der EU, Nr. L 7 vom 10. Januar 2009, S. 1-79.

68 Abkommen zwischen der Europäischen Union und den Vereinigten Staaten von Amerika über die Verarbeitung von Zahlungsverkehrsdaten und deren Übermittlung aus der Europäischen Union an die Vereinigten Staaten für die Zwecke des Programms zum Aufspüren der Finanzierung des Terrorismus. 
Freiheit, Sicherheit und Recht. ${ }^{69}$ Dieses IT-Großsystem vereint die drei wichtigsten Informationssysteme im RFSR - nämlich das Visa-Informationssystem, das Schengen-Informationssystem und EURODAC. Hiervon sind das Visa- und das Schengen-Informationssystem Gegenstand des Schengen-Besitzstands. Großbritannien und Irland sind kein Teil des Ersteren und partizipieren auch nur teilweise im Letzteren. Die ursprüngliche EURODAC-Verordnung findet in Großbritannien und Irland Anwendung. Irland wird gemäß dem grundlegenden Ansatz die Kodifizierung dieser drei Systeme abwarten, um danach zu entscheiden, ob eine Teilnahme möglich ist. Großbritannien hat jedoch offiziell die Mitteilung eingereicht, im Gesetzgebungsverfahren des Legislativpakets für das IT-Großsystem teilzunehmen. Um die ungleiche Anwendbarkeit der drei Informationssysteme für Großbritannien auszugleichen, hat der Rat der Europäischen Union eine gesonderte Erklärung verfasst, die den Schengen-Besitzstand des Visa- und Schengeninformationssystems partiell und beschränkt auf diese Systeme für Großbritannien geltend macht.

Auch Dänemark kommt in den Genuss von maßgeschneiderten Beteiligungsformen, um dem vollständigen Ausschluss im RFSR vorzubeugen. Allerdings kann dadurch meistens und wie im Fall des IT-Großsystems lediglich ein Beobachterstatus anstelle einer vollen und gleichwertigen Teilnahme Dänemarks gewährt beziehungsweise ermöglicht werden.

\section{Schlussfolgerungen}

Diesem Artikel liegt ein Analyserahmen zugrunde, der die Differenzierung bei der Arbeit - das heißt die Differenzierungsrealität - als einen pfadabhängigen Prozess mit speziellem Fokus auf die Implementierung der differenzierten Integrationsstrukturen konzeptionalisiert. Dabei spielen externe Effekte, die auf struktureller und funktioneller Ebene generiert werden, eine besondere Rolle. Die Ergebnisse der Analyse sollen im Folgenden kurz zusammengefasst werden (siehe auch Tabelle 2).

Als langfristige Konsequenz der Implementierung der Opt-Out-/Opt-In-Regime im Schengenraum wäre ein Aufbrechen der Pfadabhängigkeit dieser Differenzierungsstrukturen möglich. Der Grad der formalen Differenzierungsvolatilität ist für Großbritannien, Irland und Dänemark sowohl auf funktionaler als auch auf struktureller Ebene recht gering. Die Konditionalität der Teilnahme für Großbritannien und Irland und die obligatorische Teilnahme für Dänemark sowie der Ausschluss von den Gesetzgebungsverfahren schränken den Ermessensspielraum der Opt-Out-Staaten ein. Diese formal limitierte Differenzierungsrealität generiert relativ hohe Kosten der Schengen-Opt-Outs. Mittel- oder langfristig kann dies zu einem sogenannten ,critical juncture ‘ führen, der ein umfassendes Opt-In dieser Staaten entweder via der Implementierung der Differenzierungsstrukturen oder aufgrund einer möglichen Auflösung der Ausnahmeregelung zufolge haben kann.

Demgegenüber ist das Opt-Out-/Opt-In-Regime für Großbritannien und Irland im Titel V AEUV sehr volatil. Die Differenzierungsmuster, die durch die Implementierung dieser Strukturen entstehen, erhöhen im Allgemeinen den Nutzen der Ausnahmeregelung für Großbritannien und Irland. Demgegenüber stehen Differenzierungsmuster, die aufgrund missbräuchlicher Anwendung formaler Strukturen oder Spill-Over-Effekten zu nicht geplanten Ergebnissen führen. Dennoch ist eine Konsolidierung der Differenzierungsstrukturen trotz solcher nicht intendierter Konsequenzen zu erwarten. Die Wahlfreiheit hinsichtlich der Po-

69 Europäische Kommission: Mitteilung der Kommission. Legislativpaket zur Errichtung einer Agentur für das Betriebsmanagement von IT-Großsystemen im Bereich Freiheit, Sicherheit und Recht, KOM (2009) 292. 
litikinhalte sowie die formalen Beteiligungsmöglichkeiten in den entsprechenden Gesetzgebungsverfahren stellen den strukturellen Rahmen dafür bereit, die funktionale Anziehungskraft einzelner Maßnahmen erfolgreich wirken zu lassen. Folglich ist eine funktionelle Integration trotz struktureller Differenzierung im RFSR möglich.

Der Druck zur Auflösung der Ausnahmeregelung ist für Dänemark am höchsten, da der gänzliche Ausschluss aus dem Titel V AEUV droht. Parallelabkommen haben Dänemark bisher die Möglichkeit gegeben, mittels relativ hoher Kosten am RFSR im Rahmen von internationalem Recht teilzunehmen. Allerdings wird diese Opt-In-Möglichkeit von der Europäischen Union nur noch mit zunehmendem Unwillen gewährt. Indessen muss Dänemark seine Ausnahmeregelung nicht gänzlich auflösen, sondern hat die Möglichkeit, deren Volatilität an die des Opt-In-Regimes von Großbritannien und Irland anzugleichen. In jedem Fall ist jedoch ein Referendum in Dänemark erforderlich, welches eine substanzielle Hürde für die Veränderung der Differenzierungsrealität darstellt. 


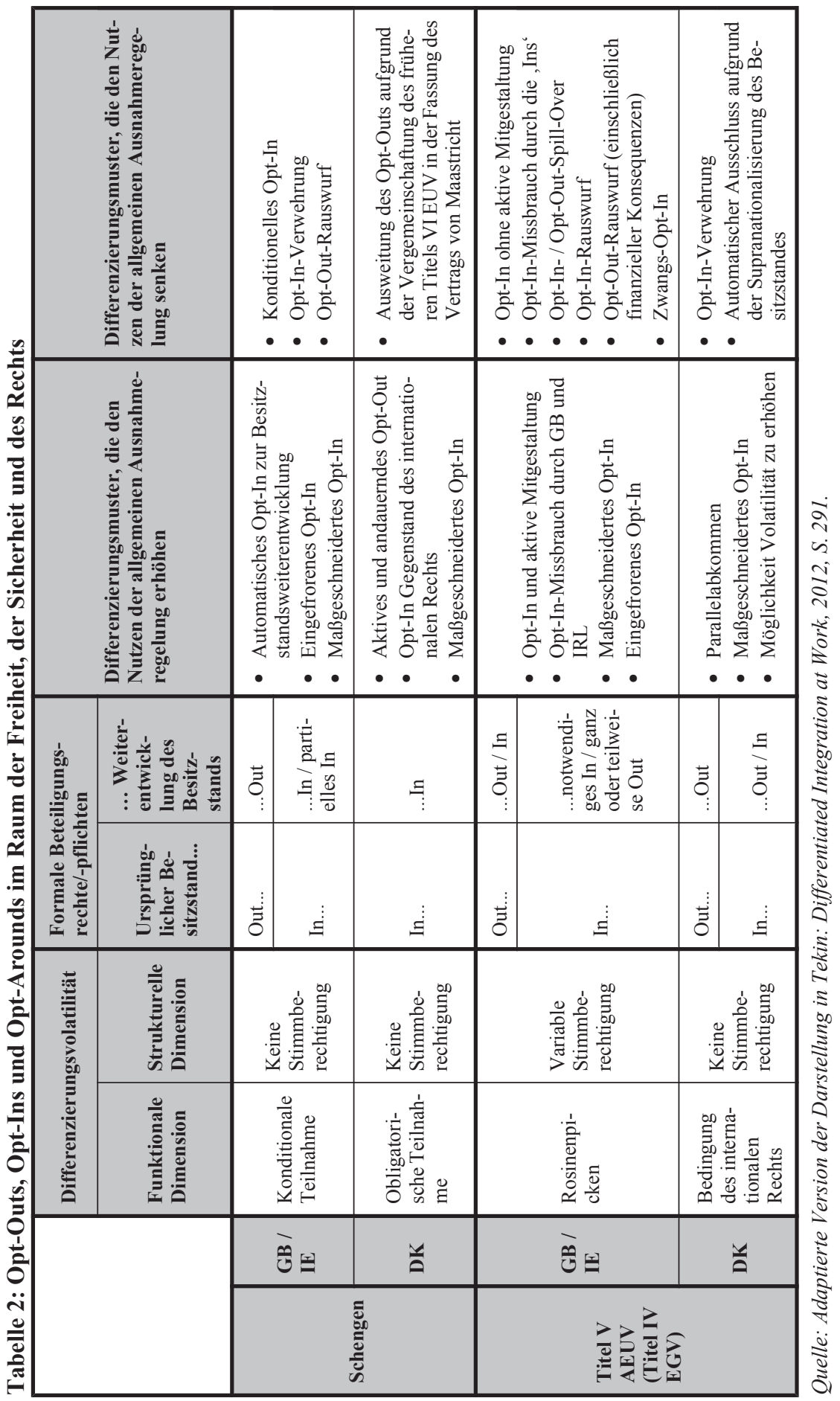

